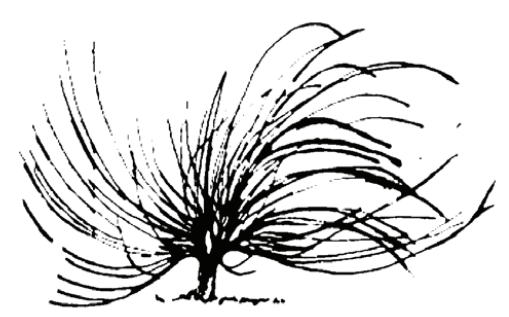

\title{
Warisata: Una Genealogía de la Educación desde el Suma Qamaña
}

\author{
María Celeste Viedma ${ }^{1}$ \\ $\mathrm{UBA} / \mathrm{CCC}$ \\ Buenos Aires, Argentina \\ mcviedma@gmail.com
}

\begin{abstract}
Resumen
Este ensayo tiene por objetivo establecer una relación de continuidad entre la pedagogía sustentada por los principales exponentes de la experiencia de la escuela Ayllu de Warisata y la actual reforma educativa impulsada por el gobierno de Evo Morales en Bolivia. Se analizan posicionamientos políticos a partir de la identificación de un enemigo, así como las dimensiones de la integralidad de la práctica pedagógica y su vínculo con los aspectos comunitario, territorial y práctico. Se trabaja principalmente con los libros escritos por Elizardo Pérez Warisata. La escuela Ayllu y por Carlos Mostajo La Taika. Teoría y práctica de la escuela ayllu, por un lado, y con la Ley de
\end{abstract}

\section{(c) (1) $(\theta)$}

Recibido: 14 de abril de 2015-Aprobado: 26 de noviembre de 2015

1 Licenciada en Sociología por la Universidad y estudiante del Profesorado secundario, normal y superior en Sociología por la Universidad de Buenos Aires. Integrante del Grupo de Estudios en Historia y Discurso del Centro Cultural de la Cooperación "Floreal Gorini”. 
Educación "Avelino Siñani - Elizardo Pérez" y el Plan Nacional de Desarrollo 2006-2011, por el otro.

Palabras clave: educación indígena, educación comunitaria, buen vivir, Bolivia, Warisata

\begin{abstract}
This essay aims to establish a relation of continuity between the pedagogy supported by the leading exponents of the experience of the ayllu school Warisata and the current educational reform driven by the government of Evo Morales in Bolivia. Political positions by the identification of an enemy are analyzed, as well as the integration of pedagogical practice and its link with the community, territorial and practical aspects. It works mainly with books written by Elizardo Pérez Warisata. The Ayllu school and Carlos Mostajo The Taika. Theory and practice of ayllu school, on one hand, and the Law on Education "Avelino Sinani - Elizardo Pérez" and the National Development Plan 2006-2011, on the other.
\end{abstract}

Keywords: indigenous education, community education, good living, Bolivia, Warisata

En la rebelión comunal, todo el pasado se concentra activamente en el presente; pero a diferencia de las épocas de quietud, donde el pasado subalterno se proyecta como presente subalternado, ahora es la acumulación del pasado insumiso el que se concentra en el presente para derrocar la mansedumbre pasada. Álvaro García Linera, La Potencia Plebeya.

¿Cuál es la propuesta pedagógica del proceso de transformación que atraviesa la sociedad boliviana bajo la dirección de Evo Morales Ayma? Tal es el interrogante con que comenzamos a pensar el presente ensayo. Formulada durante la asistencia a las clases dictadas por el equipo de Pedagogía del Profesorado en Sociología de la Universidad de Buenos Aires. La pregunta con la que iniciamos franqueó, como es lógico, cambios, revisiones, mutaciones. Algunos de ellos producto del proceso de aprendizaje recorrido en compañía del equipo dirigido por 
Carla Wainsztok, otros fruto de la lectura del Plan Nacional de Desarrollo de Bolivia en el Grupo de Estudios en Historia y Discurso, del Centro Cultural de la Cooperación. Allí donde decíamos: “¿Cuál es?”, decimos ahora ¿De dónde viene?, pues comprendemos que el ser nos inscribe siempre en una historia, que la pregunta por quiénes somos supone una noción, consciente o no, de dónde venimos, una inscripción de la propia identidad en una generación (Wainsztok, 2013). Si la educación se trata de un "encuentro entre generaciones" a través del cual la sociedad se actualiza, el ejercicio que haremos será señalar una ligazón entre procesos separados por una distancia temporal, señalando una "presencia en el presente" (Barcena, 2012). Nos preguntamos, entonces: ¿resuenan en los procesos de transformación que atraviesa actualmente la sociedad boliviana conceptos y asociaciones provenientes de otras temporalidades?

Procuramos dar respuesta a dicha interrogante a partir de la elección de una experiencia entre otras posibles: la Escuela Ayllu de Warisata. Quisiéramos presentar aquí las dimensiones de una cercanía, de una continuidad en los modos de concebir la pedagogía de la escuela Ayllu y de la actual reforma educativa impulsada por el gobierno de Evo Morales. En primer lugar, debido a que la experiencia de Warisata es ubicada entre los antecedentes del actual proceso de cambio, expresión que es el título de la misma Ley N 70 "Avelino Siñani - Elizardo Pérez", denominada así tras los nombres propios de los fundadores de la escuela Ayllu. Asimismo, se ubica en una generación que supone posicionarse políticamente en la identificación de un enemigo compartido por ambas experiencias. Señalaremos pues, en la primera parte de nuestro ensayo, los trazos de dichos posicionamientos, dando cuenta del modo en que el actual movimiento se inscribe en una tradición simbólica.

Aclaremos en este punto que trabajaremos fundamentalmente con los libros escritos por Elizardo Pérez Warisata. La escuela Ayllu y por Carlos Mostajo La Taika. Teoría y práctica de la escuela ayllu, por un lado, y con la Ley de Educación "Avelino Siñani - Elizardo Pérez" y el Plan Nacional de Desarrollo 2006-2011, por el otro. Encontramos en ambas materialidades la reverberación de una noción en particular que engloba muchas otras. En cierto modo, comprende la realidad vinculada a una totalidad diversa y compleja, una mirada holística que busca recuperar la integralidad de los procesos sociales, sea en el Vivir Bien o Suma Qamaña presente en los documentos actuales, o bien, en 
las consideraciones de Elizardo Pérez y Carlos Mostajo acerca de la "educación integral" o "educación de la vida". Las dimensiones de la interculturalidad, del "problema del indio" y del lenguaje, la vinculación con la comunidad y el territorio, las concepciones acerca del saber, la práctica, el trabajo y la espiritualidad se vinculan asimismo con este modo de comprender la realidad en forma integral. Daremos cuenta de todos ellos a lo largo de nuestro desarrollo.

Por último, señalemos que el ejercicio que nos proponemos aquí es reflexionar en torno de una inscripción en la historia y una apropiación de sentidos mostrada por los mismos discursos pedagógicos y del Vivir Bien, es decir, no es el propósito de este ensayo el análisis de aquellas relaciones discursivas no explicitadas en los documentos, sino que profundizaremos en una relación que es asumida como tal por sus autores, lo cual no quiere decir que no existan otros vínculos posibles. Comencemos, pues, con el tema que nos ocupa.

\section{Pasado y presente, nosotros y otro}

La Escuela Ayllu de Warisata fue situada por sus impulsores en el contexto de una situación de explotación de los pueblos originarios. La realidad es analizada desde categorías provenientes del marxismo latinoamericano y la práctica pedagógica considerada como resultado, a la vez, que posibilidad de transformación de las relaciones sociales de producción:

El "problema del indio" es "el de una clase en estado de servidumbre" (...) Son las relaciones de producción las que forman la base real sobre la que se alza la acción educativa, la pedagogía propiamente dicha (Mostajo, 1992:83)

Bolivia, y otras naciones como ella, en tanto no sean penetradas por la inmigración europea, son naciones indias, sometidas a minorías que explotan en su beneficio el capital humano que representan las masas indígenas (Pérez, 1992:33)

En concordancia con lo anterior, la propia práctica se inscribe en una historia de lucha, conformándose un nosotros indio y obrero, por un lado, en lucha contra el otro "enemigo feudal", la "feudal burguesía", el "imperialismo". Ambos libros presentan un recorrido por estas 
historias, donde la experiencia de Warisata es precedida por las formas de organización incaicas (Pérez, 1992), a la vez, que el "problema del indio" (Mostajo, 1992) se vincula con la temática de la apropiación de la tierra por parte de la burguesía boliviana y extranjera.

Al inscribirse Warisata en toda una tradición de resistencia y construcción, la propia práctica pedagógica debe situarse en ella y recupera los aprendizajes de dichas experiencias:

Hay que partir, pues, de estos conocimientos previos, para determinar lo que debe entenderse por sistemas de creación y aplicación de procedimientos educativos para nuestros grupos indígenas, con fines de formación de una cultura indoamericana. Ignorar la prehistoria de un grupo étnico en tales momentos es anticientifico; desde el punto de vista social y humano sería un crimen y hasta un absurdo (Pérez, 1992:33)

Vista desde fines de siglo por uno de sus protagonistas, a saber, Carlos Mostajo, la escuela ayllu tiene un carácter precursor, constituyéndose también en antecedente de los movimientos indigenistas que emergieron con posterioridad:

Algunos ignoran, de buena o mala fe, que el poderoso impulso que han cobrado los movimientos indios fue originado en las escuelas fundadas por Elizardo Pérez en la década del 30 al 40... (Mostajo, 1992:4)

Fuimos nosotros los que vencimos, pues la liquidación del feudo, en 1953, no hubiera sido posible sin el movimiento ideológico y de masas generado en Warisata (Mostajo, 1992:5)

$\mathrm{Al}$ analizar los documentos de la actualidad boliviana, encontramos también el impulso a una acción pedagógica libertaria y contraria al imperialismo:

La educación se fundamenta en las siguientes bases: (...) Es descolonizadora, liberadora, revolucionaria, anti-imperialista, despatriarcalizadora y transformadora de las estructuras económicas y sociales... (Artículo 1, Ley de Educación $\mathrm{N}^{\circ}$ 70) 
Para el neoliberalismo, los pueblos indígenas y la diversidad multiétnica y pluricultural son intrascendentes porque no contribuyen al crecimiento económico, excepto como mano de obra barata o potencial consumidor (Plan Nacional de Desarrollo, 2007:7)

El neoliberalismo o "neocolonialismo" aparece como el blanco central de las críticas y la lucha contra el mismo es puesta en serie con la resistencia al "coloniaje" y el "imperialismo". La opresión resistida por los grupos indígenas desde la colonia se actualiza hacia el siglo veintiuno bajo la forma neoliberal. La experiencia misma de Warisata es recuperada entonces desde la actualidad, como antecedente fundamental de las luchas y la organización indígena (Ministerio de Educación de Bolivia, 2012).

Desde la mirada de la actual conducción estatal boliviana, la educación indigenal constituyó un pilar fundamental en la resistencia a la opresión en tanto fue garante de la supervivencia de los saberes ancestrales combatidos por el imperialismo, a la vez, que simbolizó la lucha contra la explotación económica, política y cultural:

La importancia política de las escuelas indigenales radica en su origen como estrategia política ligada a la defensa legal de las tierras comunitarias y a la resistencia contra la expansión del latifundio (Min. Educ., 2012)

En los documentos analizados, el presente es puesto en serie junto con la experiencia de Warista y las luchas predecesoras, siendo la primera uno de los focos de resistencia de mayor envergadura dentro de las experiencias de escuelas comunitarias o escuelas rurales indígenas. Desde la perspectiva actual, la escuela ayllu permitió la transmisión y salvaguarda de propia cultura indígena. La transmisión se entiende aquí no como repetición irreflexiva, sino vinculada a la noción de generación, en relación con una filiación simbólica (Wainsztok, 2013). Nótese la consideración acerca de la escuela ayllu en la siguiente cita de Carlos Mostajo:

La escuela significaba su lazo de unión con las tradiciones históricas, con su institucionalidad, con su espiritu colectivista (Mostajo, 1992:8) 
Analizamos hasta aquí los elementos de transmisión, generación y transmisión (Wainsztok, 2013), entendidos como la filiación simbólica en la que se inscriben tanto Warisata como la conducción estatal actual de Bolivia. Vayamos ahora hacia los fundamentos pedagógicos en ellos presentes.

\section{Educación de la vida: comunidad, territorio, actividad}

Desde la perspectiva de los impulsores de la escuela Ayllu, la educación no supone únicamente la alfabetización o instrucción general de los estudiantes, sino que debe situarse en una realidad socio-económica y problematizarla:

La escuela del indio debe estar ubicada en el ambiente indio, allá donde él lucha para no desaparecer; que no debe contraerse únicamente al alfabeto sino que su función debe ser eminentemente activa y hallarse dotada de un evidente contenido social y económico (Pérez, 1992:70)

La escuela rural, conforme a la posición de Warisata, es un planteamiento cultural a la vez que económico y social, es el enfoque integral del problema del indio y de la tierra (Mostajo, 1992:8, el subrayado es nuestro)

Es frecuente en los documentos acerca de Warisata la insistencia en la necesidad de una pedagogía integral, que contemple todos los aspectos de la vida de los individuos (y la comunidad). Carlos Mostajo señala incluso que la "función integral" de la escuela puede desdoblarse en cuatro campos específicos: una integración vertical (coherencia y continuidad entre las distintas etapas de la educación desde el ciclo inicial hasta la formación de educadores), integración horizontal (con el ambiente, las relaciones sociales que la circundan), integración activa (forma de actividad tripe entre la escuela, el taller y el sembrío), integración económico-social y cultural, referida a que la escuela desarrolla sus actividades en el contexto de la lucha de clases (Mostajo, 1992).

Destaquemos primeramente que integralidad no supone abstracción o simplicidad sino, por el contrario, se trata de una mirada holística que complejiza la realidad, procurando aprehenderla en toda su diversidad, a la vez, que se opone a lo que Pablo Pineau denomina positivismo 
pedagógico, que establece la cientificidad como único criterio de validación y privilegia los procesos intelectuales por sobre los afectivos, corporales, artísticos (Pineau, 2007). La siguiente cita es ilustrativa de lo que queremos expresar:

El pedagogismo es dogmático, estrecho y circunscripto: la vida es amplia, móvil, atrayente, diversa (Pérez, 1940 - citado en Mostajo, 1992:84)

Habría, entonces, dos tipos de pedagogía: aquella capaz de formar para la liberación y aquella que reproduce las condiciones de existencia. Educación integral significa asimismo en estrecha relación con las condiciones sociales, una educación situada en un territorio y con una historia específica, donde educar significa por lo tanto, formar conciencia revolucionaria:

No es una preparación para la vida, sino la vida misma, la vida resultante de la división de clases, de la desigualdad y de la injusticia, la vida que exige imperiosamente un cambio, la vida creadora de conciencia y aptitud de lucha, en lugar de ser una adaptación conformista a modalidades actuales (Mostajo, 1992:89, el subrayado es del autor)

Al ser "la vida misma", la escuela no se interrumpe, no tiene "período lectivo" y "vacaciones", sino que la labor es permanente:

No se trataba de un mero establecimiento educacional con un periodo lectivo tras el cual se suspenden las labores; sino que era toda una empresa de carácter económico social (Pérez, 1992:115)

Nos interesa señalar aquí la centralidad que adquiera la noción de la vida dentro de la propuesta pedagógica de Warisata. Se trata de una ligazón con las luchas indígenas del pasado para ir hacia el futuro, una revolución que suponga no sólo la transformación de las relaciones económicas sino también y, quizás más importante, la conquista de una liberación política y cultural, que involucre todos los aspectos de la vida. 
Las dimensiones cultural, política y económica se encuentran en este paradigma profundamente ligadas y no pueden separarse unas de otras:

Warisata se propuso enfocar el problema indio no únicamente desde el plano educativo, sino contemplando todos los aspectos económico-sociales y culturales que le son inherentes. Este propósito le daba a Warisata una dimensión integral, y en este caso, el término adquiere su exacto valor semántico, pues que engloba la totalidad de los aspectos en que se desarrolla la vida del indio (Mostajo, 1992:17)

Es en este punto que quisiéramos introducir las consideraciones acerca del Suma Qamaña o Vivir Bien, recuperadas por el actual gobierno boliviano. Lo que se enarbola allí es una concepción del desarrollo que exceda lo meramente económico, postulando "una visión cosmocéntrica que supera los contenidos etnocéntricos tradicionales del desarrollo" (PND, 2007:8). El concepto es recuperado de las culturas originarias y se refiere a que el cosmos es "el centro de la dinámica espiritual, material, económica, social y política de las comunidades andino amazónicas" (PND, ídem). En el aspecto educativo, la educación para Vivir Bien supone también una formación integral, donde integralidad significa la convivencia armónica entre culturas diversas:

Es educación de la vida y en la vida, para Vivir Bien. Desarrolla una formación integral que promueve la realización de la identidad, afectividad, espiritualidad y subjetividad de las personas y comunidades; es vivir en armonía con la Madre Tierra y en comunidad entre los seres humanos (Artículo 3, Ley de Educación $\mathrm{N}^{\mathrm{o}} 70$ )

Se trata de una forma de educación que se vincula con la vida en sus dimensiones material, espiritual y afectiva (Min. Educ., 2012), recordándonos una frase producida desde otras latitudes y tiempos acerca de "los efectos de los afectos" (Iglesias, 2004). En su artículo primero, la Ley de Educación citada anteriormente sintetiza los contenidos que desarrollaremos a lo largo del presente ensayo: 
El sistema educativo se fundamenta en una educación abierta, humanista, cientifica, técnica y tecnológica, productiva, territorial, teórica y práctica, liberadora y revolucionaria, crítica y solidaria (Artículo 1, Ley de Educación $\mathrm{N}^{\mathrm{o}} 70$, el subrayado es nuestro)

Tres concepciones que resultan fundamentales para comprender la profundidad de la noción de "educación de la vida" o "pedagogía integral": comunidad, territorio-ruralidad y productividad. Volvamos, pues, a Warisata.

Lo central de la educación indigenal es que, para ser transformadora, debe desarrollarse en el seno de una comunidad. La escuela no es, desde esta perspectiva, una institución de encierro, aislada del resto de la sociedad, sino que se imbrica en las relaciones comunitarias a la vez que se inscribe, como se señaló al comienzo de este ensayo, en una historia de luchas y resistencias:

Más allá de la escuela está la escuela. El vasto mundo de nuestro ayllu es el verdadero claustro de la escuela; el indio nos lo enseña (Pérez, 1992:171)

La escuela asume la forma de organización del Parlamento Amauta, "forma revitalizada de la secular ulaka y del ayllu aymaro-quechua" (Pérez, 1992:116) y se vincula con toda la comunidad, con el ayllu, recuperando las formas de organización indígenas históricas, fundamento sociológico de Warisata (Mostajo, 1992). Esto significa que el territorio, su condición rural, asume una importancia fundamental y que cada escuela será diversa de acuerdo con el territorio en el que se encuentre:

La escuela rural no puede ser la misma que la escuela urbana; los sistemas pedagógicos deberán contemplar la naturaleza especial de los problemas que a cada nivel corresponden (Mostajo, 1992:84)

Tiende la escuela a la formación de hombres prácticos capaces de bastarse a sí mismos, sobre manera, dentro y para el radio económico en que deben desarrollar su existencia. Esto quiere decir que el indio será educado para vivir en el agro, cultivarlo, impulsarlo, enriquecerlo, llevarlo a la expresión de máxima 
riqueza. Continúase de esta manera una tradición socialista que comienza desde los inkas (Pérez, 1992:169)

La importancia otorgada al territorio y a la comunidad obedece también a la vinculación de la escuela con la producción, donde "escuela integral" y "escuela productiva" designan "un mismo contenido" (Mostajo, 1992:87). He aquí otro aspecto de la pedagogía integral liberadora de Warisata que quisiéramos señalar: la manera en que teoría y práctica se imbrican en su pedagogía, donde la actividad productiva y su potencialidad creadora son parte de la tarea pedagógica y el saber, el conocimiento, no se concibe, como en la educación colonial, separado de la práctica, sino puesto a su servicio:

Los egresados de la Escuela nos serían enciclopedistas, como los bachilleres urbanos, pero sin duda responderían más adecuadamente a las exigencias de la vida social (Mostajo, 1992:96)

El mismo sostenimiento material de la escuela se realizaba desde sus integrantes en vinculación con la comunidad, lo que permitía asimismo la perpetuación de las culturas y formas de organización indígenas:

El producto cosechado, agrícola o industrial, se destina al sostenimiento de la escuela, que como centro de actividad de la comunidad está encaminado a formar una nueva sociedad indígena basándola en sus sobrevivientes instituciones culturales (Pérez, 1992:124).

La importancia atribuida a la producción se acompaña de una consideración de la importancia de la formación espiritual tanto de estudiantes como maestros:

La escuela era una forja real del espíritu: el mismo maestro iba a educarse en su ambiente, a adquirir reciedumbre y esperanza (Pérez, 1992:116)

Volvamos ahora al presente, veamos cuántas de estas nociones resuenan en la propuesta pedagógica del actual gobierno boliviano. La educación en Bolivia: 
Es productiva y territorial, orientada a la producción intelectual y material, al trabajo creador y a la relación armónica de los sistemas de vida y las comunidades humanas en la Madre Tierra, fortaleciendo la gestión territorial de las naciones y pueblos indígenas originario campesinos, las comunidades interculturales $y$ afro bolivianas (Artículo 3, Ley de Educación $\mathrm{N}^{\circ} 70$ )

El fortalecimiento de la gestión territorial se realiza a partir de la organización en tres niveles (Central, Departamental y Autonómico), a la vez, que se promueve la participación de las comunidades locales diversas en la gestión y la planificación educativas (Artículos 90 y 91). El enfoque comunitario y territorial no se establece únicamente en el ámbito educativo sino en todos los aspectos de gobierno, fortaleciendo la autogestión de las comunidades y reconociendo las relaciones culturales que se establecen en los diversos territorios (PND, 2007). En el aspecto escolar, como complementario a la Participación Comunitaria se implementa el Currículo Regionalizado, que contempla la adaptación curricular de acuerdo con la localización de cada escuela en particular, destacándose el siguiente punto respecto de las escuelas indígenas:

Las naciones y pueblos indigenas originarios campesinos desarrollan procesos educativos productivos comunitarios, acorde a sus vocaciones productivas del contexto territorial (Artículo 70, Ley de Educación $N^{\circ} 70$, el subrayado es nuestro)

Se fomenta la construcción de conocimiento crítico, vinculado con la realidad social en la que el proceso educativo se ve inmerso (Artículo 3), se revalorizan los saberes ancestrales de las comunidades originarias y la práctica pedagógica recupera los saberes y experiencias que los estudiantes traen consigo previo el ingreso a la institución ${ }^{2}$. Dichos saberes se formalizan a partir de la Certificación de Competencias (Artículo 83). La vinculación entre conocimiento y producción no se realiza únicamente respecto de los pueblos originarios, sino que teoría y práctica se entretejen en el proceso educativo:

2 La consideración del alumno como un sujeto que trae a la escuela aprendizajes se opone también a la pedagogía positivista que supone estudiantes rasos, sin conocimientos ni aptitudes previos a su ingreso en la institución escolar, fuente de todo conocimiento válido (Pineau, 2007). 
La organización curricular establece los mecanismos de articulación entre la teoría y la práctica educativa, se expresa en el currículo base de carácter intercultural, los currículos regionalizados y diversificados de carácter intracultural que en su complementariedad, garantizan la unidad e integridad del Sistema Educativo Plurinacional, así como el respeto a la diversidad cultural y lingüistica de Bolivia (Artículo 69, Ley de Educación $\mathrm{N}^{\mathrm{o}} 70$ )

Antes de profundizar en las nociones de intra e interculturalidad (presentes en la cita anterior), volvamos brevemente a Warisata en la búsqueda de sus resonancias. Quisiéramos señalar como último aspecto de nuestro análisis, la potencialidad de que sus integrantes atribuyen a las escuelas indígenas para la conformación de una cultura netamente boliviana, expresión de la diversidad de tradiciones que la conforman. En este punto, la lengua cobra una importancia fundamental como elemento de las culturas y potencialidad liberadora:

Sostenemos que el indio debe conservar su idioma, y no solamente esto, sino que la escuela debe contribuir a purificarlo y enriquecerlo, porque el idioma es la expresión mental más característica de una cultura (...) Nuestra escuela es, pues, bilingüe; y lo es, porque los idiomas maternos no deben desaparecer, con el objeto de que concurra a ese gran proceso de decantación idiomática que dará con el tiempo un idioma estrictamente boliviano (Pérez, 1953 - citado en Mostajo, 1992:120)

¿Qué podemos hacer con la cita anterior sino ponerla en juego con la transformación reciente de Bolivia en Estado Plurinacional? La educación actualmente en Bolivia "es intracultural, intercultural y plurilingüe en todo el sistema educativo" (Artículo 1, Ley de Educación $\mathrm{N}^{\circ} 70$ ). La interculturalidad es central incluso para el desarrollo económico, político y cultural al promover la interacción y complementariedad entre culturas (PND, 2007). Se trata de reafirmar la "unidad en la diversidad" (Artículo 3), lo que supone la recuperación y fortalecimiento al interior de las culturas originarias (intraculturalidad) y "el desarrollo de la interrelación e interacción de conocimientos, saberes, ciencias y tecnología propios de cada cultura con otras 
culturas, que fortalece la identidad propia y la interacción en igualdad de condiciones" (Artículo 5). La educación también será plurilingüe e iniciará en la lengua materna, variando de acuerdo con la prevalencia de la misma en la comunidad (Artículo 7). El horizonte es la conformación de una identidad nacional que se enriquezca a partir de la convivencia y el respeto entre culturas diversas:

De esta manera, la diversidad se convierte en un factor de cohesión social y no de separación y enfrentamiento (PND, 2007:17)

\section{A modo de conclusión}

A lo largo del presente ensayo, pusimos en serie la experiencia de Warisata y las transformaciones recientes de la sociedad boliviana. Procuramos señalar el modo en que desde el presente se vuelve al pasado, vinculando la práctica pedagógica a la integralidad de la vida, por un lado, y atendiendo al modo en que lo comunitario, territorial y práctico se hacen presentes en unos y otros documentos, por el otro. Trazamos, como lo señalamos al comienzo, un camino posible entre múltiples, deteniéndonos en una apropiación que es explícitamente asumida por los actuales discursos pedagógicos y del Vivir Bien.

Desplegamos las dimensiones de dicha cercanía a partir de la inscripción del actual movimiento en una filiación simbólica en la que la escuela ayllu ocupa un lugar privilegiado. Desde la actualidad, la "educación del indio" constituye un elemento central en la resistencia frente al neoliberalismo o "neocolonialismo" en la medida en que permitió la transmisión de los saberes ancestrales de las comunidades, entendida no como una repetición irreflexiva sino vinculada a una tradición. Encontramos, pues, una recuperación y una puesta en valor de la misma experiencia de Warisata, que la inscribe como una de las experiencias de mayor importancia en dichos procesos.

Asimismo, nos detuvimos en los puntos de contacto que pueden observarse entre la concepción del Suma Qamaña o Vivir Bien y la "educación integral" o "educación de la vida" enarbolada por los exponentes de Warisata. Se trata de una comprensión de la realidad a partir de su carácter de totalidad compleja y diversa, una mirada holística que tal vez nos resulte extraña luego de los tiempos del "fin de la historia", al tiempo que nos propone recuperar un modo de pensar que 
reponga la preocupación por integralidad de los procesos sociales. Una integralidad que no diluye, no obstante, la diversidad y multiplicidad de procesos. También se hacen presentes las consideraciones de índole espiritual y la importancia de incorporar dicha dimensión a los procesos de enseñanza y aprendizaje.

El abordaje del "problema del indio", del lenguaje y la interculturalidad son elementos también característicos de la educación del Estado Plurinacional, al igual que la vinculación del proceso pedagógico a la comunidad y al territorio. Al problematizar la totalidad social en que los estudiantes se ven inmersos, la comunidad y el territorio de los que la escuela es parte y en los que tienen lugar no pueden quedar "puertas afuera" de las aulas, sino que permean todo el proceso educativo. Este debe hacer lugar a las diversas lenguas y culturas que habitan las aulas bolivianas. Lo mismo puede decirse acerca de la práctica, el trabajo y su relación con el saber. Si la escuela se vinculará con la comunidad, no puede resultar ajena a la actividad productiva sino que debe procurar transformarla en actividad creadora. El conocimiento no puede desarrollarse separado de la práctica, ni tampoco de las relaciones sociales en las que se ve inmerso. ¿Resuenan, entonces, en los procesos de transformación que atraviesa actualmente la sociedad boliviana conceptos y asociaciones provenientes de la Escuela Ayllu? No podemos sino responder afirmativamente a nuestro interrogante.

Reflexionemos. Lo que intentamos realizar es la puntualización de una relación, el señalamiento de resonancias, de aprendizajes de las luchas del pasado que reverberan en el presente. Quien escribe considera que este tipo de ejercicios supone realizar una "genealogía de la memoria histórica de nuestros países" (Quintar, 2005) poniéndonos a nosotros mismos, como científicos sociales en la misma línea espacio-temporal, incluyéndonos en ese nosotros que "se abre entre los sujetos" (Quintar, 2005) y que no está suturado, sino que se reinventa permanentemente. Una reinvención que puede enriquecerse aún más si procuramos tejer un diálogo entre nuestras prácticas científica y pedagógica, entre nuestra posición como investigadores y como docentes. Las dimensiones analizadas en este recorrido procuran ser una contribución a dicho encuentro. Un encuentro amoroso entre los múltiples nosotros que nos habitan, una escucha a las experiencias de lucha de nuestros pueblos que nos permita sostener coherentemente con Mariátegui que "no queremos, ciertamente, que el socialismo sea en América 
calco y copia. Debe ser creación heroica. Tenemos que dar vida, con nuestra propia realidad, en nuestro propio lenguaje, al socialismo indo-americano. He aquí una misión digna de una generación nueva" (Mariátegui, 1971:249).

\section{Referencias bibliográficas}

Barcena, F. (2012) Entre generaciones. Notas sobre la educación en la filiación del tiempo. Rosario: Homo Sapiens.

García Linera, A. (2009) La Potencia Plebeya. Acción colectiva e identidades indigenas, obreras y populares en Bolivia. Bogotá: Siglo del Hombre Editores y CLACSO.

Mariategui, J. C. (1971) Aniversario y balance. En Ideología y política. Lima: Biblioteca Amauta.

Ministerio de Educación (2012) Currículo Base del Sistema Educativo Plurinacional. Documento de trabajo. Revista Educación y ciudad $N^{o} 22$, páginas 57-80. Bogotá: IDEP.

Ministerio de Planificación del Desarrollo (2007) Plan Nacional de Desarrollo. "Bolivia digna, soberana, productiva y democrática para Vivir Bien”. La Paz: Artes Gráficas Sagitario.

Mostajo, C. S. (1992) La Taika. Teoría y Práctica de la Escuela Ayllu. La Paz: G.U.M.

Pérez, E. (1992) Warisata. La escuela ayllu. La Paz: Talleres gráficos Hisbol.

Pineau, P. (2007) La escuela como máquina de educar. Buenos Aires: Paidos.

Quintar, E. (2005) La esperanza como práctica. La práctica como ámbito de construcción de futuro. Revista Interamericana de Educación de Adultos $N^{\circ}$, páginas 250-253. México: CREFAL.

Wainsztok, C. (2013) El concepto de comunidad en nuestras aulas de la CELAC. Solidaridad Global $N^{\circ} 22$, páginas 34-41. Villa María: Universidad Nacional de Villa María. 